\title{
The extraction of parameters from final state interactions
}

\author{
G. Fäldt ${ }^{1, *}$ and C. Wilkin², 田 \\ ${ }^{1}$ Department of Physics and Astronomy, Uppsala University, Box 516, 75120 Uppsala, Sweden \\ ${ }^{2}$ Physics and Astronomy Department, UCL, Gower Street, London WC1E 6BT, United Kingdom
}

(Dated: July 27, 2021)

\begin{abstract}
It is argued that final state enhancements in production reactions at large momentum transfers, such as $p p \rightarrow K^{+} \Lambda p$, are primarily sensitive to the position of a virtual bound state pole in the $\Lambda p$ system rather than the $\Lambda p$ scattering length and effective range. These arguments are supported by a study of the dispersion relation derived to describe such processes as a function of the cut-off energy. This shows that the position of the virtual bound state is independent of the cut-off energy.
\end{abstract}

PACS numbers: 13.75.-n, 13.75.Cs, 13.75.Ev

\section{INTRODUCTION}

It is difficult to study directly the scattering of some elementary particles at low energies, especially if they are unstable and neutral, as is the case for the $\Lambda$ hyperon. Nevertheless, a few low statistics measurements have been made of the $\Lambda p$ cross sections in hydrogen bubble chambers for center-of-mass (c.m.) momenta above about $60 \mathrm{MeV} / c$ [1, 2].

An alternative approach is to look at the $\Lambda p$ system through a Final State Interaction (FSI) in a production experiment, such as $p p \rightarrow K^{+} \Lambda p$. By studying purely the kaon in an inclusive measurement, it is possible to deduce directly the missing mass $m_{X}$ in the reaction and, for an excitation energy in the $\Lambda p$ system $Q=\left(m_{X}-m_{\Lambda}-m_{p}\right) c^{2}<77 \mathrm{MeV}$, conservation laws of strong interactions ensure that the unobserved system $X=\Lambda+p$. The direct fitting of enhancements in such data [3, 4], as described in Sec. II] shows that there is a pole in the $\Lambda p$ scattering amplitude that corresponds to a virtual (antibound) state in this system. However, the general pursuit in the literature has not been into the extraction of the properties of this pole but rather aimed at the determination of the $\Lambda p$ scattering length $(a)$ and the effective range $(r)$, which depend in a model-dependent way also on the behavior at much higher values of $Q$.

In an effort to avoid the high $Q$ problem, where the assumption that the data are dominated by $S$ waves in the $\Lambda p$ system is highly questionable, analyticity of the amplitudes has been exploited to give estimates in terms of a dispersion integral over a finite range in $Q[5,6]$. This method, which is described in Sec. III, provides theoretical estimates of the uncertainties arising from the cut in $Q$ and, to a lesser extent, those associated with the meson production operator. It has been used extensively to extract information from exclusive $p p \rightarrow K^{+} \Lambda p$ data taken by the COSY-TOF collaboration [7, 8]. However, once again the stress has been placed on the determination of

\footnotetext{
*E-mail: goran.faldt@physics.uu.se

${ }^{\dagger}$ E-mail: c.wilkin@ucl.ac.uk
}

the scattering length [, $[, 6]$.

The numerical evaluations of the dispersion integral presented in Sec. IV are largely consistent with the findings reported in the theoretical papers [5, 6]. With a cut-off at $Q_{\max }=40 \mathrm{MeV}$, the scattering length is reduced by about $0.3 \mathrm{fm}$ but with important changes also in the effective range. The main point of the current paper is to show that, although $a$ and $r$ depend significantly on $Q_{\max }$, the position of the nearby virtual state pole remains fixed when $Q_{\max }$ is reduced from infinity to $40 \mathrm{MeV}$. Any uncertainty in determining the pole position arising from the choice of $Q_{\max }$ is negligible compared to the experimental errors. It is mainly this feature of the $\Lambda p$ interaction that is determined by the final state enhancement rather than the scattering length and effective range separately. The dependence of the pole position on the structure of the meson production operator also appears to be much weaker.

Though the arguments given in this paper are couched mainly in terms of the $p p \rightarrow K^{+} \Lambda p$ reaction, the principal results are valid much more widely. Final state interactions in high momentum transfer reactions are more sensitive to the positions of nearby bound or virtual state poles in the scattering amplitudes rather than to asymptotic observables, such as the scattering length.

As summarized in the conclusions of Sec. V] we have investigated the effects of truncating the dispersion integral expression for the $S$-wave phase shift. It was already clear from the work reported in Refs. [5, 6 ] that the resulting scattering length would be modified and that this had to be taken into account. However, it was not evident from these works that the position of the nearby pole in the scattering amplitude was stable to changes in the cut-off parameter.

It is clear from its derivation that in the dispersion integral approach it is assumed that the differential cross section for a high momentum transfer production reaction, such as $p p \rightarrow K^{+} \Lambda p$, is determined by the absolute square of the $\Lambda p$ Jost function, i.e., that the meson production operator is of zero range. Though it is quite plausible that short-distance effects dominate, changing the range of the production operator would lead one to deduce a different value of the scattering length. How- 
ever, the position of the $\Lambda p$ virtual state pole is little affected by the range in the production operator, and this is also discussed in Sec. V.

\section{DIRECT FITTING OF FSI ENHANCEMENTS}

Experiments to measure inclusive kaon production in proton-proton collisions were carried out at Saclay [3] and more recently by the HIRES collaboration at COSY [4]. They both showed an enormous enhancement just above the $\Lambda p$ threshold that is associated with the $\Lambda p$ final state interaction (FSI).

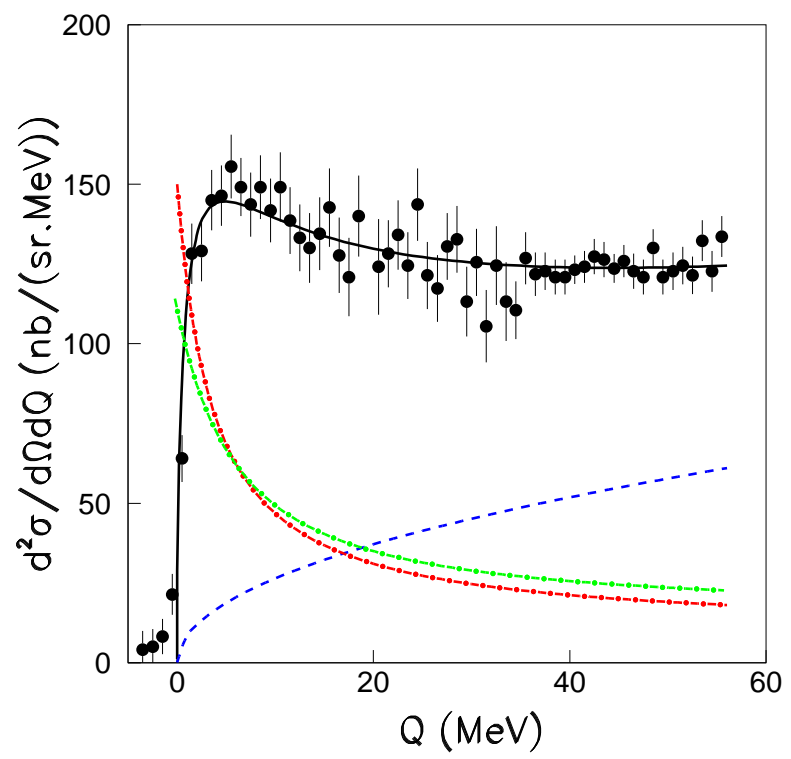

FIG. 1: (color online) Missing-mass spectrum of the reaction $p p \rightarrow K^{+}\{X=\Lambda p\}$ measured at $\theta_{K}=0^{\circ}$ by the COSYHIRES collaboration at $T_{p}=1.953 \mathrm{GeV}$ [4]. The (black) solid line represents the description of Eq. (1), including the $\Lambda p$ FSI generated by the Jost function of Eq. (2). The experimental fit takes into account the missing-mass resolution with $\sigma=$ $0.84 \mathrm{MeV}$, but this is not important for the present discussion and is not shown. The (blue) dashed line represents the shape of the $p p \rightarrow K^{+} \Lambda p$ phase space distribution, whereas the (red) chain curve is the shape of the Jost representation of the $\Lambda p$ FSI. The arbitrarily normalized FSI function of Eq.(9) used in the analysis of the COSY-TOF experimental data [8] is shown by the less steep (green) chain curve.

The fit to the COSY-HIRES data in Fig. 1 used the factorization

$$
\frac{\mathrm{d}^{2} \sigma}{\mathrm{d} \Omega_{K} \mathrm{~d} m_{X}}=A\left(m_{X}\right) \Phi_{3}=|\mathcal{M}|^{2} \Phi_{3}|J(k)|^{-2},
$$

where $\Phi_{3}$ reflects the three-body phase space and $\mathcal{M}$ is a production amplitude that is assumed to vary very slowly with the $\Lambda p$ relative momentum $k$. The crucial postulate is that the production operator is of zero range such that the FSI can be expressed in terms of the Jost function $J(k)$.

The COSY-HIRES data [4] are well described by a one-pole Jost function $J(k)$, where

$$
J(k)=\frac{k-i \alpha}{k+i \beta}
$$

The parameters deduced from the experimental data are $\alpha=(-0.31 \pm 0.03) \mathrm{fm}^{-1}$ and $\beta=(1.21 \pm 0.14) \mathrm{fm}^{-1}$, where the sign of $\alpha$ is chosen such that there is no bound state in the $\Lambda p$ system and only statistical uncertainties are quoted. The Jost function of Eq. (2) corresponds to the Bargmann potential [9], for which the effective range expansion

$$
k \cot \delta(k) \approx-\frac{1}{a}+\frac{1}{2} r k^{2},
$$

is exact. Here $\delta(k)$ is the $S$-wave $\Lambda p$ phase shift. The experimental results are [4]

$a=\frac{\alpha+\beta}{\alpha \beta}=-2.43 \pm 0.17 \mathrm{fm}, r=\frac{2}{\alpha+\beta}=2.21 \pm 0.16 \mathrm{fm}$.

The immediate complication in the analysis is that there are two $\Lambda p S$ waves, corresponding to the singlet and triplet combinations of the hyperon and proton spins. The quoted parameters are therefore some average over the two spin combinations, with unknown relative weights that depend on the production mechanism and could vary with both the beam energy and the kaon angle.

Although the data of Fig. 1 are clearly sensitive to the position of the nearby pole, i.e., the value of $\alpha$, the COSY-HIRES authors stressed instead the values obtained for the scattering length and effective range [4], and this bias has continued in the more sophisticated analysis discussed in the next section.

\section{DISPERSION RELATION ESTIMATES}

The direct fitting approach has been rightly criticized because it assumes that, even at high excess energy $Q$, the $\Lambda p$ system remains in the $S$ wave [5, 6]. There are other complications at higher $Q$ because of the coupling to the $\Sigma N$ channel as well as distortions of the matrix element $\mathcal{M}$ due to the excitation of $N^{\star}$ isobars. In an effort to avoid these problems, analyticity properties of the production amplitude were exploited to yield a dispersion integral. Though its full evaluation would require an integration over $Q$ to infinity, the authors integrate only over a finite range, $0 \leq Q \leq Q_{\max }$, and then make theoretical estimates of the possible contributions from regions beyond $Q_{\max }[5,6]$.

The starting point for the dispersion approach is similar to that of the direct fitting procedure, which is that 
the $p p \rightarrow K^{+}\{\Lambda p\}$ double-differential cross section is proportional to the phase space multiplied by the reciprocal of the absolute square of the $\Lambda p$ Jost function. Having made this assumption, it is possible to generalize the arguments given in the standard texts [10, 11] to express the $S$-wave phase shift in terms of two dispersion integrals. The integral up to $Q_{\max }$ is over the logarithm of the Jost function and that beyond $Q_{\max }$ depends upon the phase shift itself. In the approach adopted in Refs. [5, 6], the phase shift is approximated by the first finite integral.

In the cut-off approximation, the $S$-wave phase shift is given by [5],

$$
\begin{gathered}
\frac{\delta(k)}{k}=-\frac{1}{2 \pi} \sqrt{\frac{m_{\min }}{m_{\mathrm{red}}}} \mathbf{P} \int_{m_{\min }^{2}}^{m_{\max }^{2}} \mathrm{~d} \mu^{2} \sqrt{\frac{m_{\max }^{2}-m_{X}^{2}}{m_{\max }^{2}-\mu^{2}}} \times \\
\frac{1}{\sqrt{\mu^{2}-m_{\min }^{2}}\left(\mu^{2}-m_{X}^{2}\right)} \log \{A(\mu)\} .
\end{gathered}
$$

Here $m_{\text {red }}$ is the reduced hyperon-proton mass and the minimum missing mass is given by $m_{\min }=m_{p}+m_{\Lambda}$. In order to be consistent with the potential description, the relation between $k$ and the missing mass has been taken as the non-relativistic $m_{X}=m_{\text {min }}+k^{2} / 2 m_{\text {red }}$. The choice of the cut-off parameter $m_{\max }=m_{\min }+Q_{\max } / c^{2}$ is clearly subjective. Ideally it should be as large as possible, subject to the $\Lambda p$ system still being in an $S$-wave. It was argued that for $\Lambda$ production the best compromise would be achieved by taking $Q_{\max }=40 \mathrm{MeV}$ [5], which is well below the $\Sigma N$ thresholds.

It should be noted that the integral vanishes if the argument of the logarithm in Eq. (5) is constant so that, by subtracting the integrand with $A(\mu)$ replaced by $A\left(m_{X}\right)$, it is possible to replace the principal value $(\mathbf{P})$ integral by a Riemann integral:

$$
\begin{gathered}
\frac{\delta(k)}{k}=-\frac{1}{2 \pi} \sqrt{\frac{m_{\min }}{m_{\mathrm{red}}}} \int_{m_{\min }^{2}}^{m_{\max }^{2}} \mathrm{~d} \mu^{2} \sqrt{\frac{m_{\max }^{2}-m_{X}^{2}}{m_{\max }^{2}-\mu^{2}}} \times \\
\frac{1}{\sqrt{\mu^{2}-m_{\min }^{2}}\left(\mu^{2}-m_{X}^{2}\right)} \log \left\{\frac{A(\mu)}{A\left(m_{X}\right)}\right\},
\end{gathered}
$$

where the singularity at $\mu=m_{X}$ in the denominator is canceled by the zero from the logarithm.

The $k$ on the left hand side of Eq. (6) is the $\Lambda p$ c.m. momentum corresponding to the missing mass $m_{X}$ so that the estimate of the scattering length is obtained by taking the limit $m_{X} \rightarrow m_{\text {min }}$, i.e., $a=-\lim _{k \rightarrow 0}\{\delta(k) / k\}$. However, by evaluating Eq. (6) at a range of values of $k$ it is straightforward to extract approximations for the effective range or higher terms in a $k^{2}$ expansion.

The numerical evaluation is facilitated if the integration variable is changed to

$$
\mu^{2}=m_{\min }^{2} \cos ^{2} \theta+m_{\max }^{2} \sin ^{2} \theta
$$

which leads to

$$
\frac{\delta(k)}{k}=-\frac{1}{\pi} \sqrt{\frac{m_{\min }\left(m_{\max }^{2}-m_{X}^{2}\right)}{m_{\mathrm{red}}}} \int_{0}^{\pi / 2} \mathrm{~d} \theta \frac{\log \left\{A(\mu) / A\left(m_{X}\right)\right\}}{\left(\mu^{2}-m_{X}^{2}\right)} .
$$

It should be noted that, if one takes the Jost function of Eq. (2) as input for $A$, then Eq. (8) yields the correct Bargmann values of the scattering length and effective range, but only in the limit where $Q_{\max } \rightarrow \infty$. The pole is then at the "correct" place of $k=i \alpha$.

The stability of the pole position is, however, a more general result. Below threshold, $m_{X}<m_{\min }$, the dispersion denominator in Eq. (8) does not vanish and the integration over the $\log \{A(\mu)\}$ term remains finite. There remains the second integral proportional to $A\left(m_{X}\right)$ and, if this has a pole, as it does for example in Eq. (2), then $\delta \rightarrow-i \infty$ at this point and the scattering amplitude diverges. A virtual state pole would therefore remain in a fixed position, independent of the cut-off.

\section{NUMERICAL STUDIES}

Values of the approximations to the scattering length and effective range for the Jost function input of Eq. (2) were obtained through the numerical evaluation of Eq. (6) as functions of the cut-off energy $Q_{\max }$. As shown in Fig. 2, for large $Q_{\max }$ these approach the asymptotic values of $a=-2.43$ and $r=2.21$ [4]. The variation of the scattering length with $Q_{\max }$ is also consistent with previous estimates [5], changing by about $0.3 \mathrm{fm}$ from its asymptotic value by $Q_{\max }=40 \mathrm{MeV}$.

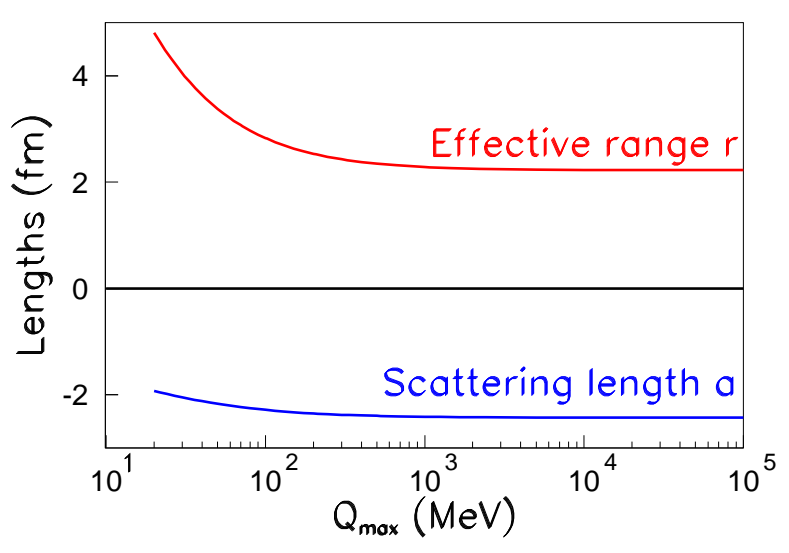

FIG. 2: (color online) Evaluations of the approximations to the scattering length and effective range for the Jost function of Eq. (2) as functions of $Q_{\max }$. The asymptotic parameters used in this study, $\alpha=-0.307 \mathrm{fm}^{-1}$ and $\beta=1.212 \mathrm{fm}^{-1}$, were those found by the COSY-HIRES collaboration [4].

Of much greater interest is the variation of the Jost parameters deduced by fitting the effective range expan- 
sion of Eq. (3) to the values extracted for the scattering length and effective range. Though, as shown in Fig. 3 . at $Q_{\max }=40 \mathrm{MeV} \beta$ deviates from its asymptotic value by almost a third, the change in $\alpha$ is barely $1 \%$. Variations of this size arise when finding the position of the nearby pole by using the truncated effective range expansion of Eq. (3) and the stability of $\alpha$ is confirmed by finding the pole position after keeping the $k^{4}$ term in the effective range expansion. The result should also not be understood as implying that the value of $\alpha$ is determined to $1 \%$ since the input function, which was not changed in the evaluation, has significant experimental uncertainty. Rather the result signals that the error in $\alpha$ induced by employing the truncated effective range expansion with different choices of $Q_{\max }$ is negligible compared to the other uncertainties.

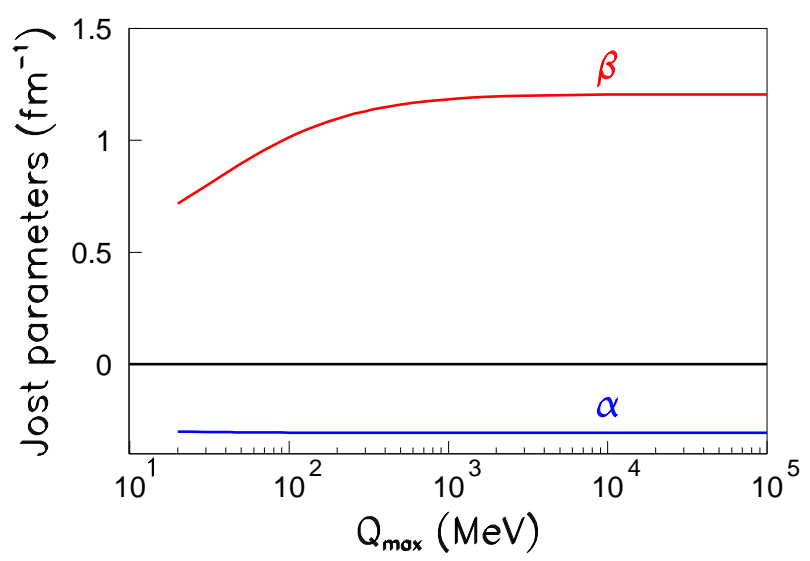

FIG. 3: (color online) Evaluations on the basis of the truncated effective range expansion of Eq. (3) of the approximations to the pole parameters for the Jost function of Eq. (2) as functions of $Q_{\max }$. The asymptotic parameters used in this exercise, $\alpha=-0.307 \mathrm{fm}^{-1}$ and $\beta=1.212 \mathrm{fm}^{-1}$, were those found by the COSY-HIRES collaboration [4].

If, instead of the Jost function of Eq. (2), one takes

$$
A\left(m_{X}\right) \propto \exp \left[C_{1}^{2} /\left(m_{X}^{2}-C_{2}^{2}\right)\right]
$$

then the integral of Eq. (6) can be evaluated to give an analytic approximation for the scattering length [5] (and a more complicated one for the effective range). This was the form assumed for the extraction of the scattering length from the COSY-TOF data, with parameter values of $C_{1}=348 \mathrm{MeV} / c^{2}$ and $C_{2}=2039.2 \mathrm{MeV} / c^{2}$ [8]. It must be noted that Eq. (9) should not be used below threshold because it has the wrong analytic structure, with an essential singularity rather than a simple pole. In such a case the stability of the virtual state pole to changes in the cut-off energy cannot be proved a priori.

Nevertheless, as with the Jost input of Eq. (2), the estimates of the scattering length and effective range change steadily with $Q_{\max }$ but the nearby pole, determined from the truncated effective range expansion, remains far more stable, only changing from $\alpha=-0.421 \mathrm{fm}^{-1}$ at $Q_{\max }=100 \mathrm{MeV}$ to $\alpha=-0.417 \mathrm{fm}^{-1}$ at $Q_{\max }=$ $40 \mathrm{MeV}$. This value is somewhat different from the $\alpha=-0.31 \pm 0.04 \mathrm{fm}^{-1}$ found by the COSY-HIRES collaboration [4] but systematic uncertainties are hard to quantify. Any deviations between the two experimental results, which seem to be already indicated by the FSI functions shown in Fig. 1, could be due to disparities between the spin-average COSY-TOF [7, 8] and COSYHIRES [4] experimental data rather than the different methodologies in the fitting processes. The larger value for $|\alpha|$ found at COSY-TOF is consistent with the less steep enhancement factor shown in the curves of Fig. 1 .

\section{CONCLUSIONS}

The amplitude for a production reaction such as $p p \rightarrow$ $K^{+} \Lambda p$ at large momentum transfers is sensitive to the $\Lambda p$ wave function at short distances. By taking the meson production operator to be of zero range, it is assumed that the amplitude depends on the $\Lambda p$ Jost function. In the standard approach, as adopted for example by the COSY-HIRES collaboration [4], the parameters of the Jost function are obtained from a direct fit to the experimental data. Values of the scattering length and effective range are then deduced from these parameters.

There are several problems in implementing such a direct fit approach. Since the beam energy is finite, the data do not extend beyond some limited range of excitation energy and other mechanisms are likely to become important at large $Q$. Of immediate concern has to be the assumption that the $\Lambda p$ system remains in the $S$-wave for arbitrarily large values of $Q$. In an attempt to avoid these problems, approximations for the $S$-wave phase shift were obtained through a dispersion integral over a finite range of $0 \leqslant Q \leqslant Q_{\max }$ and estimates were made of the error in truncating the integration at $Q_{\max }[5,[6]$. It has been argued that the best compromise for the $p p \rightarrow K^{+} \Lambda p$ reaction would be to take $Q_{\max }=40 \mathrm{MeV}$ and this is the value used in the analysis of the COSY-TOF data [7, 8].

The principal result of our analysis is that, even if one accepts all of the assumptions made in Refs. [5, 6], it is clear that it is the position of the nearby pole that is completely stable to changes in $Q_{\max }$ rather than the value of the scattering length, which would therefore require model-dependent corrections or error estimates. There are, of course, other sources of error in the evaluation of the pole position but these are largely independent of the choice of $Q_{\max }$.

The theoretical papers of Refs. [5, 6] have pointed out that it is possible to study the spin dependence of the $S$ wave $\Lambda p$ interaction through measurements of final state enhancements produced by polarized proton beams. This important program has been pursued with the COSY- 
TOF experimental data [7, 8]. However, the COSYHIRES data were taken with an unpolarized beam in the forward direction where any analyzing power would, in any case, vanish [4]. One can therefore only compare the COSY-TOF and COSY-HIRES unpolarized data. Though these were taken at similar beam energies, there could in principle still be differences arising from the different angular coverage in the two experiments if the $\Lambda p$ scattering parameters had a large spin dependence. Even if we neglect such a possibility, the significance of the difference between the values of $\alpha(\mathrm{TOF}) \approx-0.42 \mathrm{fm}^{-1}$ and $\alpha($ HIRES $)=-0.31 \pm 0.04 \mathrm{fm}^{-1}$ found in the two experiments must depend upon a careful assessment of the systematic uncertainties.

The current understanding of the low energy $\Lambda$-nucleon interaction has been nicely summarized in a recent review [13]. All the potentials discussed there generate nearby $S$-wave virtual bound states in both the spinsinglet and -triplet $\Lambda$-nucleon systems, with typically $\alpha$ (singlet $) \approx-0.28 \mathrm{fm}^{-1}$ and $\alpha$ (triplet $) \approx-0.38 \mathrm{fm}^{-1}$. These are not very different to the values derived from the COSY-HIRES and COSY-TOF data but these experimental results correspond to spin averages with unknown weights.

Both the direct fitting and the dispersion relation approach assume that the meson production operator in these high momentum transfer reactions is of very short range. The validity of this assumption will, of course, depend on both the form of the operator and the shortrange $r$ behavior of the two-body wave function. In the case of $p p \rightarrow \pi^{+} p n$, the short-range repulsion in the $p n$ system will diminish the importance of the behavior at $r=0$ [12]. To avoid this problem, the variation of the Paris 14] $S$-wave spin-triplet $p n$ wave function with $k$ was studied at $r=1.05 \mathrm{fm}[15]$. Though the pole position, which is fixed by the deuteron binding energy, is independent of the value chosen for $r$, the momentum dependence of the wave function at $r=1.05 \mathrm{fm}$ is weaker than that given by the Jost function. In the language of the Bargmann potential, the value of $\beta$ would have been increased and so the scattering length deduced from the Jost function would be in error, though the value of $\alpha$ would be correct.

It was suggested several years ago that the positions of the nearby two-body poles seem to govern the energy dependence of the total meson production cross section near threshold [16] and this approach has been used to describe the $p p \rightarrow K^{+} \Lambda p$ total cross section at low energies [17]. If the pole corresponds to a true bound rather than a virtual one it is even possible to determine the normalization from the residue at the pole. This allows one to estimate the total cross section for $n p$ spin-triplet production in $p p \rightarrow p n \pi^{+}$in terms of that for $p p \rightarrow d \pi^{+}[18$ ].

We have largely ignored any questions of experimental errors and, with perfect data, even a small range in $Q$ near threshold might be sufficient to allow extrapolation to the pole. This is naturally a very naive limit but it does offer a simple explanation as to why it is the value of $\alpha$ extracted from the dispersion integrals of Eq. (6) that is almost independent of $Q_{\max }$.

The inspiration of this work came from discussions with the authors of Refs. [6], 8], and [4]. The constructive suggestions made by A. Gasparyan and J. Haidenbauer were much appreciated.
[1] G. Alexander et al., Phys. Rev. 173, 1452 (1968).

[2] B. Sechi-Zorn, B. Kehoe, J. Twitty, and R. A. Burnstein, Phys. Rev. 175, 1735 (1968).

[3] R. Siebert et al., Nucl. Phys. A567, 819 (1994).

[4] A. Budzanowski et al., Phys. Lett. B687, 31 (2010).

[5] A. Gasparyan, J. Haidenbauer, C. Hanhart, and J. Speth, Phys. Rev. C 69, 034006 (2004).

[6] A. Gasparyan, J. Haidenbauer, and C. Hanhart, Phys. Rev. C 72, 034006 (2005).

[7] F. Hauenstein, PhD thesis, University of ErlangenNürnberg (2014).

[8] F. Hauenstein et al., arXiv:1607.04783 (2016).

[9] V. Bargmann, Phys. Rev. 75, 301 (1949).

[10] M. L. Goldberger and K. M. Watson, Collision Theory
(Wiley, New York, 1964).

[11] R. G. Newton, Scattering Theory of Waves and Particles, (Springer-Verlag, New York, 1982).

[12] V. R. Pandharipande, (private communication) (1996).

[13] A. Gal, E. V. Hungerford, and D. J. Millener, Rev. Mod. Phys. 88, 035004 (2016).

[14] M. Lacombe, B. Loiseau, J. M. Richard, R. Vinh Mau, J. Côté, P. Pirès, and R. de Tourreil, Phys. Rev. C 21, 861 (1980).

[15] G. Fäldt and C. Wilkin, Phys. Rev. C 56, 2067 (1997).

[16] G. Fäldt and C. Wilkin, Z. Phys. A 357, 241 (1997).

[17] S. Sewerin et al., Phys. Rev. Lett. 83, 682 (1999).

[18] G. Fäldt and C. Wilkin, Phys. Lett. B382, 209 (1996). 